\title{
Nonlocal continuum modeling and molecular dynamics simulation of torsional vibration of carbon nanotubes
}

\author{
F. Khademolhosseini, A.S. Phani, A. Nojeh and R.K.N.D. Rajapakse
}

\begin{abstract}
This work investigates the size-effects in the dynamic torsional response of single walled carbon nanotubes (SWCNTs) by developing a modified nonlocal continuum shell model. The purpose is to facilitate the design of devices based on CNT torsion by providing a simple, accurate and efficient continuum model that can predict the frequency of torsional vibrations and the propagation speed of torsional waves. To this end, dispersion relations of torsional waves are obtained from the proposed nonlocal model and compared to classical models. It is seen that the classical and nonlocal models predict non-dispersive and dispersive behavior, respectively. Molecular dynamics (MD) simulations of torsional vibrations of $(6,6)$ and $(10,10)$ SWCNTs are also performed, the results of which are compared with the classical and nonlocal models and used to extract consistent values of the nonlocal elasticity constant. The superiority and accuracy of the nonlocal elasticity model in predicting the size-dependent dynamic torsional response of SWCNTs is demonstrated.
\end{abstract}

Index Terms - Torsional oscillator, carbon nanotube, molecular dynamics, nonlocal elasticity.

\section{INTRODUCTION}

C Carbon nanotubes (CNTs) have attracted much attention from scientists and engineers because of their relevance to a wide range of applications in several branches of engineering. Over the past decade torsional paddle oscillators have been developed, which use carbon nanotubes as torsional spring elements. Papadakis et al. [1] built resonant paddle oscillators with multi-walled carbon nanotubes as torsional springs. Hall et al. [2] built a paddle oscillator by suspending a 400-nm gold paddle on a SWCNT less than a micrometer in length. A schematic representation of such a device is shown in Fig. 1. Through quasi-static actuation of the paddle by applying a voltage bias between the base plate and the gold paddle, they induced torsion in the SWCNT and measured its torsional

Manuscript submitted March 25, 2010. This work was supported by a grant from the Natural Sciences and Engineering Research Council of Canada.

F. Khademolhosseini and A.S. Phani are with the Department of Mechanical Engineering, University of British Columbia, Vancouver, V6T 1Z4, Canada (e-mail:khadem@interchange.ubc.ca, srikanth@mech.ubc.ca).

A. Nojeh is with the Department of Electrical and Computer Engineering, University of British Columbia, Vancouver, V6T 1Z4, Canada (e-mail: anojeh@ece.ubc.ca).

R.K.N.D Rajapakse was with the Department of Mechanical Engineering, University of British Columbia, Vancouver, V6T 1Z4, Canada. He is now with the Faculty of Applied Sciences, Simon Fraser University, Burnaby, Canada V5A 1S6 (Corresponding author e-mail: rajapakse@mech.ubc.ca, Phone: 1-778-782-3826; Fax:1-778-782-5802). properties such as the average shear modulus [2]. By applying an AC signal of variable frequency, they observed the resonance behavior of the SWCNT-based torsional paddle oscillator and measured resonance frequencies around $400 \mathrm{kHz}$ and quality factors ranging from 450 to 1150 [3]. Use of carbon nanotubes as torsional elements in devices such as electromechanical switches, torsional delay elements, electromechanical filters, single-molecule torsional oscillators (CNT without suspended paddle) and AFM tips can also be contemplated. In all of the aforementioned devices, the dynamic torsional response of the CNT spring element underpins the overall performance of the device; thus the characterization and modeling of dynamic torsional properties of CNTs could substantially inform the design process. Earlier studies employed multi-scale modeling of CNT oscillators that require significant computational time [4,5]. A continuum modeling approach, which accounts for size-effects, can provide a much more computationally efficient alternative.

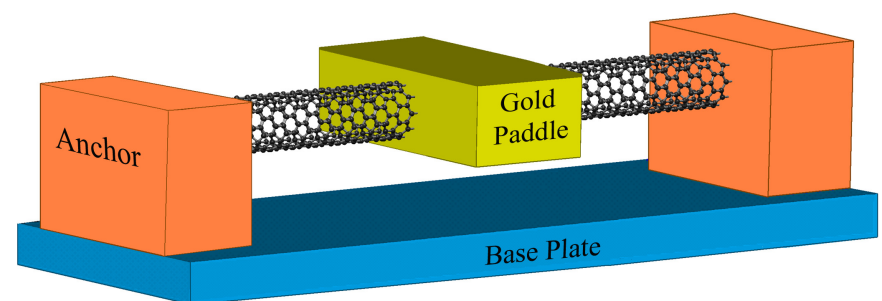

Fig. 1. Schematic of SWCNT torsional paddle oscillator/electromechanical switch.

Among the various approaches used for the characterization of CNT properties [6-8], continuum modeling has generated much interest due to computational efficiency. At the nanoscale, however, the dimensions of a system are comparable to the inter-atomic or inter-molecular spacing of that system, and the material can no longer be modeled as a continuum. Modified continuum models have been proposed to overcome the limitations of classical continuum mechanics, among which, models based on Eringen's theory of nonlocal elasticity [9] have proven effective in capturing the size-dependent mechanical response of CNTs [10-12]. It should be noted that continuum models need to be first verified against more accurate, atomistic methods such as molecular dynamics simulations before applying to analyze nanoscale systems. 
Classical continuum beam and shell models are unable to accurately predict the dynamic response of CNTs when the deformation wavelengths become comparable to the internal characteristic length of CNT systems, i.e., the carbon-carbon bond length [13-15]. As an example, classical shell models predict non-dispersive behavior for axi-symmetric torsional waves traveling along a CNT, while MD simulations show dispersive behavior [16]. To overcome this deficiency of classical continuum models in modeling the torsional response of CNTs, Wang and Varadan [12] proposed a nonlocal elastic model based on the Flugge shell theory. Unlike the classical Flugge shell model, the model proposed in Ref. [12] was able to predict the dispersive behavior of axi-symmetric torsional waves in a CNT in the high-frequency region. In this model, the magnitude of dispersion depended on the value of the nonlocal constant, which had to be determined for CNTs. This task was undertaken by $\mathrm{Hu}$ et al. [16] who compared MD simulation results for the phase velocity of axi-symmetric torsional waves propagating in a CNT with Wang's model and estimated the nonlocal constant of CNTs to be in the range 0.2-0.6.

The MD simulations reported in [16] require further investigation. First, the data points simulated are in the low-frequency (long-wavelength) region where the dispersive behavior is less present and size effects are insignificant. Hence the classical and nonlocal models do not differ significantly in the long-wavelength/low-frequency regime. In fact, only one data point has been simulated for the high-frequency region in [16] and the estimation of the nonlocal constant is based on that one point. Secondly, the simulation procedure entails the harmonic excitation of one end of the CNT and the observation of the time it takes the excited wave of a given frequency to travel the distance between two specific points on the CNT. Although this is a possible way of measuring the phase velocity, it is severely limited by the phonon band-structure of the CNT, which might contain band-gaps i.e., frequency bands in which waves do not propagate. This would make it a challenge to choose the frequency of the sinusoidal wave, excited at one end of the CNT (in fact, this could be the reason why there are so few data points in the high-frequency region of the simulations in [16]). Furthermore, there is no real justification that the excited wave consists of only one frequency, as it could actually be a wave-packet consisting of several different frequencies.

Based on the above observations, the present study proposes to investigate the free torsional vibrations (standing waves) of CNTs instead of torsional waves to develop a set of results that is more useful for practical applications. Note that natural modes are standing waves arising from the superposition of two propagating waves of same frequency travelling in the opposite direction. That is, standing waves are discrete points on the dispersion curve for propagating waves. Thus, they are able to show the general dispersive behavior of propagating waves as well as providing the following benefits:

-Studying the free torsional vibrations of CNTs, allows us to accurately simulate different mode-shapes of short and long wavelengths, providing us with a rich dataset, especially in the high-frequency regions of the dispersion curve where the difference between the classical and nonlocal models is expected to be more apparent. This can result in an accurate estimation of the nonlocal elasticity constant of CNTs.

-In the present work, the MD simulation outputs are vibration frequencies, which can be used to calculate both the group and the phase velocities of propagating waves. The group velocity is of special interest as it controls the energy flow in the CNT.

-It is possible to visualize the mode-shape over several hundred free vibration time periods and ensure that only one specific natural frequency has been excited.

This paper is organized as follows. Section II introduces the dispersion relations based on the classical and nonlocal elasticity models. In section III MD simulation results are presented for the axi-symmetric torsional vibrations of $(10,10)$ and $(6,6)$ armchair nanotubes. The natural frequencies from MD simulations are then compared to those obtained from the nonlocal model and an estimate for the nonlocal constant is derived. The phase and group velocities of MD simulations are then compared to classical and nonlocal models and the superiority of the nonlocal model in predicting the dispersive behavior seen in MD simulations is established.

\section{Classical and Nonlocal Dispersion RELATIONS}

Assuming a CNT can be modeled as a cylindrical shell with mean radius $a$ and uniform thickness $h$ the modified nonlocal form of the differential equation which governs the torsional vibrations / wave propagation is [16]:

$$
\frac{E h}{2(1+v)}\left(1+\frac{h^{2}}{12 a^{2}}\right) \frac{d^{2} v}{d x^{2}}=\left(1-\xi \nabla_{R}^{2}\right)(\rho h \ddot{v}),
$$

where $v$ is the circumferential displacement, $E$ is the Young's modulus of the CNT, $v$ is the Poisson's ratio for CNTs, $\xi=\left(e_{0} d\right)^{2}$ is the nonlocal parameter, $e_{0}$ is the nonlocal constant to be determined for CNTs and $d$ is the internal characteristic length of the system, i.e., the C-C bond-length. Now taking into account that $h^{2} / a^{2}<<1$ and introducing the shear wave speed $c=\sqrt{G / \rho}$, the above equation can be simplified to:

$\ddot{\phi}-\xi \frac{d^{2} \ddot{\phi}}{d x^{2}}-c^{2} \frac{d^{2} \phi}{d x^{2}}=0$,

where $\phi$ is the rotation of the cross-section at $x$ around the CNT axis. This is the nonlocal form of the governing differential equation for the torsional vibrations of a cylindrical shell. In the absence of nonlocal effects, $\xi=0$ and the above equation reduces to its classical form, $\ddot{\phi}-c^{2}\left(d^{2} \phi / d x^{2}\right)=0$ [17].

\section{A. Propagating Wave Solution}

Consider a plane torsional wave of the form $\phi=\phi_{0} e^{i(k x-\omega t)}$ propagating along the axis of the cylindrical shell, where $\phi_{0}$ is the amplitude, $i$ is the imaginary unit of complex numbers, 
$k=2 \pi / \lambda$ is the wave-number, $\lambda$ is the wavelength and $\omega$ is the angular frequency of the propagating wave. The following identities can be verified for the plane wave:

$\ddot{\phi}=-\omega^{2} \phi, \quad \frac{d^{2} \phi}{d x^{2}}=-k^{2} \phi, \quad \frac{d^{2} \ddot{\phi}}{d x^{2}}=k^{2} \omega^{2} \phi$.

Substituting the above relations into equation (2) the following characteristic equation governing the wave-numberfrequency relation (or dispersion) is obtained:

$\left(-\omega^{2}-\xi \omega^{2} k^{2}+c^{2} k^{2}\right) \phi=0$.

Thus, for a torsional wave propagating in a cylindrical shell, the dispersion relation based on nonlocal elasticity is:

$$
\omega^{2}=\frac{c^{2} k^{2}}{1+\xi k^{2}}=\frac{c^{2} k^{2}}{1+e_{0}^{2} d^{2} k^{2}} .
$$

Once again, in the absence of nonlocal effects $(\xi=0)$, the above equation reduces to its classical (nondispersive) form, $\omega^{2}=c^{2} k^{2}$.

It is instructive to examine and compare the group and phase velocities of propagating waves based on the frequency relations obtained above for both the nonlocal and classical solutions.

\section{Classical Solution}

The phase velocity, $C_{p}$ is the ratio of the angular frequency, $\omega$, to the corresponding wave-number, $k$. The group velocity, $C_{g}$, is the ratio of infinitesimal changes in $\omega$ and $k$ (or the local slope of the $\omega-k$ curve). The classical dispersion relation $\left(\omega^{2}=c^{2} k^{2}\right)$ is used to obtain phase and group velocities:

$C_{p}=\frac{\omega}{k}=c, C_{g}=\frac{d \omega}{d k}=c$.

It is seen that in the classical solution the phase and group velocities are equal to the shear speed of sound and are constant for all wave-numbers / frequencies, indicating no dispersion.

\section{Nonlocal Solution}

Here the nonlocal dispersion relation seen in equation (5) is used to find group and phase velocities. The phase velocity is:

$$
C_{p}=\frac{\omega}{k}=\frac{c}{\left(1+\xi k^{2}\right)^{1 / 2}}=\frac{c}{\left(1+e_{0}^{2} d^{2} k^{2}\right)^{1 / 2}},
$$

and the group velocity is:

$$
C_{g}=\frac{d \omega}{d k}=\frac{c}{\left(1+\xi k^{2}\right)^{3 / 2}}=\frac{c}{\left(1+e_{0}^{2} d^{2} k^{2}\right)^{3 / 2}} .
$$

It is seen that in the nonlocal solution, the group and phase velocities depend on the frequency/wave-number of the propagating wave, indicating dispersion. The strength of dispersion is governed by the positive nonlocal parameter, $\xi$. The phase and group velocities are smaller in the nonlocal case than the classical value $c$ and they decrease as the frequency of the waves increase. With increasing frequency, group velocity decreases at a faster rate compared to phase velocity. It is obvious to verify that classical results (equation (6)) ensue from the nonlocal results in the limiting case of $\xi=0$.

\section{B. Standing Wave Solution}

When covering MD simulations of the dynamic torsional response of CNTs, it will be seen that it is much easier to simulate and analyze the free vibrations (standing or non-propagating waves) of a CNT in order to see the real dispersion behavior. Therefore, the free torsional vibrations of pined-pined CNTs are simulated here and the corresponding natural frequencies are studied to calculate group and phase velocities and analyze the dispersion behavior. If $\phi(x, t)$ is the rotation of the cross-section of a nanotube at a distance $x$ from the support at time $t$, the pinned-pinned boundary conditions state that:

$\phi(0, t)=0, \phi(l, t)=0$,

where $l$ is the length of the nanotube.

For each simulation, the nanotube is pre-deformed to a natural mode-shape at $t=0$ and released with zero initial velocity. Thus the initial conditions (at $t=0$ ) are:

$\phi(x, 0)=A \sin \frac{k^{\prime} \pi x}{l}, k^{\prime}=1,2, \ldots$

$\frac{d}{d t} \phi(x, 0)=0$,

where $k^{\prime}$ is the non-dimensional wave-number (corresponding to the number of half waves along the length of the nanotube) related to $k$ via $k^{\prime}=k l / \pi$ and $l$ is the length of the nanotube.

If $\omega$ is the angular frequency of the vibration it can be shown that:

$\phi(x, t)=A \sin \frac{k^{\prime} \pi x}{l} \cos \omega t$,

where equation (11) is a solution of the governing differential equation (2) and satisfies the boundary and initial conditions of (9) and (10). Substituting this solution into equation (2) the following relation is obtained:

$\left[\left(l^{2}+\xi k^{\prime 2} \pi^{2}\right) \omega^{2}-k^{\prime 2} \pi^{2} c^{2}\right] \phi=0$.

The dispersion relation is found by equating the term in the brackets in the above equation to zero: 


$$
\omega^{2}=\frac{c^{2}\left(\frac{k^{\prime 2} \pi^{2}}{l^{2}}\right)}{1+\xi\left(\frac{k^{\prime 2} \pi^{2}}{l^{2}}\right)}=\frac{c^{2}\left(\frac{k^{\prime 2} \pi^{2}}{l^{2}}\right)}{1+e_{0}^{2} d^{2}\left(\frac{k^{\prime 2} \pi^{2}}{l^{2}}\right)} .
$$

For comparison the classical dispersion relation is obtained by setting the nonlocal parameter to zero:

$$
\omega^{2}=c^{2}\left(\frac{k^{\prime 2} \pi^{2}}{l^{2}}\right) .
$$

Taking note that $k^{\prime}$ is related to the wave-number $k\left(k=k^{\prime} \pi / l\right)$, it is seen that, as expected, the dispersion relations of equations (13) and (14) are the same as the dispersion relations derived in equations (5) and (7), respectively. So the classical and nonlocal group and phase velocities derived in equations (6) to (8) hold for the case of free vibrations as well. This was anticipated from the beginning since free vibrations can be thought of as the superposition of two propagating waves of the same velocity and amplitude traveling in opposite directions.

\section{Comparison of Classical and Nonlocal Models}

Based on equations (7) and (14) classical models predict that for torsional waves (or torsional vibrations) in a cylindrical shell there is a linear relationship between the wave-number (or mode-shape) and the corresponding natural frequency. Classical models also predict that the phase and group velocities of torsional waves in a cylindrical shell are constant, independent of the wave-number and equal to the shear speed of sound.

In contrast to classical models, the nonlocal models derived in equations (5) and (13) show a nonlinear relation between the wave-number of a torsional wave and its natural frequency. For higher wave-numbers with smaller wave lengths, nonlocal models predict natural frequencies that are smaller than those predicted by classical models. This increased compliance of nonlocal models is due to the additional deformation mechanisms allowed in accounting for the total strain energy.

A useful analogy to recall is the higher compliance of the Timoshenko beam model compared to the Euler-Bernoulli beam model. It is well known that the Timoshenko model predicts lower values for natural frequencies and is indeed unavoidable for describing vibrations of thick beams where shear deformation is important, while the Euler-Bernoulli beam theory does not account for shear deformation. Even for thin beams, shear corrections become important at higher frequencies.

Nonlocal models predict that torsional waves in cylindrical shells are dispersive; as the wave-number increases the corresponding phase and group velocities decrease. The highest phase and group velocities obtained from the nonlocal model correspond to the first mode shape or the smallest wave-number and are equal to the shear speed of sound. It is important to note from the nonlocal model that the magnitude of dispersion depends on the value of the nonlocal parameter, $\xi$.

It is useful to investigate the general behavior of the nonlocal model, compare its nonlinearity to the linear classical model and quantify the relative errors that occur if classical models are used instead of nonlocal models. The nonlocal relation between $\omega$ and $\mathrm{k}$ in equation (6) can be expanded as a Taylor series expansion of the following form:

$$
\begin{aligned}
& \omega_{(k)}^{2}=\frac{c^{2} k^{2}}{1+\xi k^{2}} \rightarrow \omega_{(k)}=c k\left(1+\xi k^{2}\right)^{-1 / 2} \\
& \Rightarrow \omega_{(k)}=c k+\frac{-1 / 2}{1 !} c \xi k^{3}+\frac{3 / 4}{2 !} c \xi^{2} k^{5}+\cdots .
\end{aligned}
$$

It is seen that a polynomial describing the $\omega-k$ behavior should only have odd powers of $k$. The above expression can be used to find the general behavior of group and phase velocities:

$$
\begin{aligned}
& C_{g}=\frac{d \omega_{(k)}}{d k}=c+\frac{-3 / 2}{1 !} c \xi k^{2}+\frac{15 / 4}{2 !} c \xi^{2} k^{4}+\cdots \\
& C_{p}=\frac{\omega_{(k)}}{k}=c+\frac{-1 / 2}{1 !} c \xi k^{2}+\frac{3 / 4}{2 !} c \xi^{2} k^{4}+\cdots .
\end{aligned}
$$

It is seen that polynomials describing the $C_{p}-k$ and $C_{g^{-}}-k$ behavior should only have even powers of $k$ and are quadratic in nature. The relative errors in calculating $\omega(k), C_{g}$ and $C_{p}$ using the classical model are:

$$
\begin{aligned}
\operatorname{error}\left(\omega_{(k)}\right) & =\frac{\omega_{(k), \text { classical }}-\omega_{(k), \text { nonlocal }}}{\omega_{(k), \text { classical }}} \\
& =\frac{1 / 2}{1 !} \xi k^{2}-\frac{3 / 4}{2 !} \xi^{2} k^{4}+\cdots, \\
\operatorname{error}\left(C_{g}\right) & =\frac{C_{g, \text { classical }}-C_{g, \text { nonlocal }}}{C_{g, \text { classical }}} \\
& =\frac{3 / 2}{1 !} \xi k^{2}-\frac{15 / 4}{2 !} \xi^{2} k^{4}+\cdots, \\
\operatorname{error}\left(C_{p}\right) & =\frac{C_{p, \text { classical }}-C_{p, \text { nonlocal }}}{C_{g, \text { classical }}} \\
& =\frac{1 / 2}{1 !} \xi k^{2}-\frac{3 / 4}{2 !} \xi^{2} k^{4}+\cdots,
\end{aligned}
$$

It is observed that the relative error is larger at higher wave-numbers. As an example, for a mode-shape with a wave-number of $1.3 \times 10^{10}$, corresponding to a wavelength of 4.8 angstroms, using a nonlocal constant value of $e_{0}=0.2$ and an interatomic spacing of $d=1.41$ angstroms $\left(\xi=e_{0}^{2} d^{2}\right)$, based on equation (17) the relative errors in calculating $\omega(k), C_{g}$ and $C_{p}$ using the classical model are $7 \%, 20 \%$ and $7 \%$, respectively and the nonlocal values of $\omega(k), C_{g}$ and $C_{p}$ are equal to $93 \%$, $80 \%$ and $93 \%$ of the corresponding classical values, 
respectively. For larger values of the nonlocal constant, the errors could be even more significant.
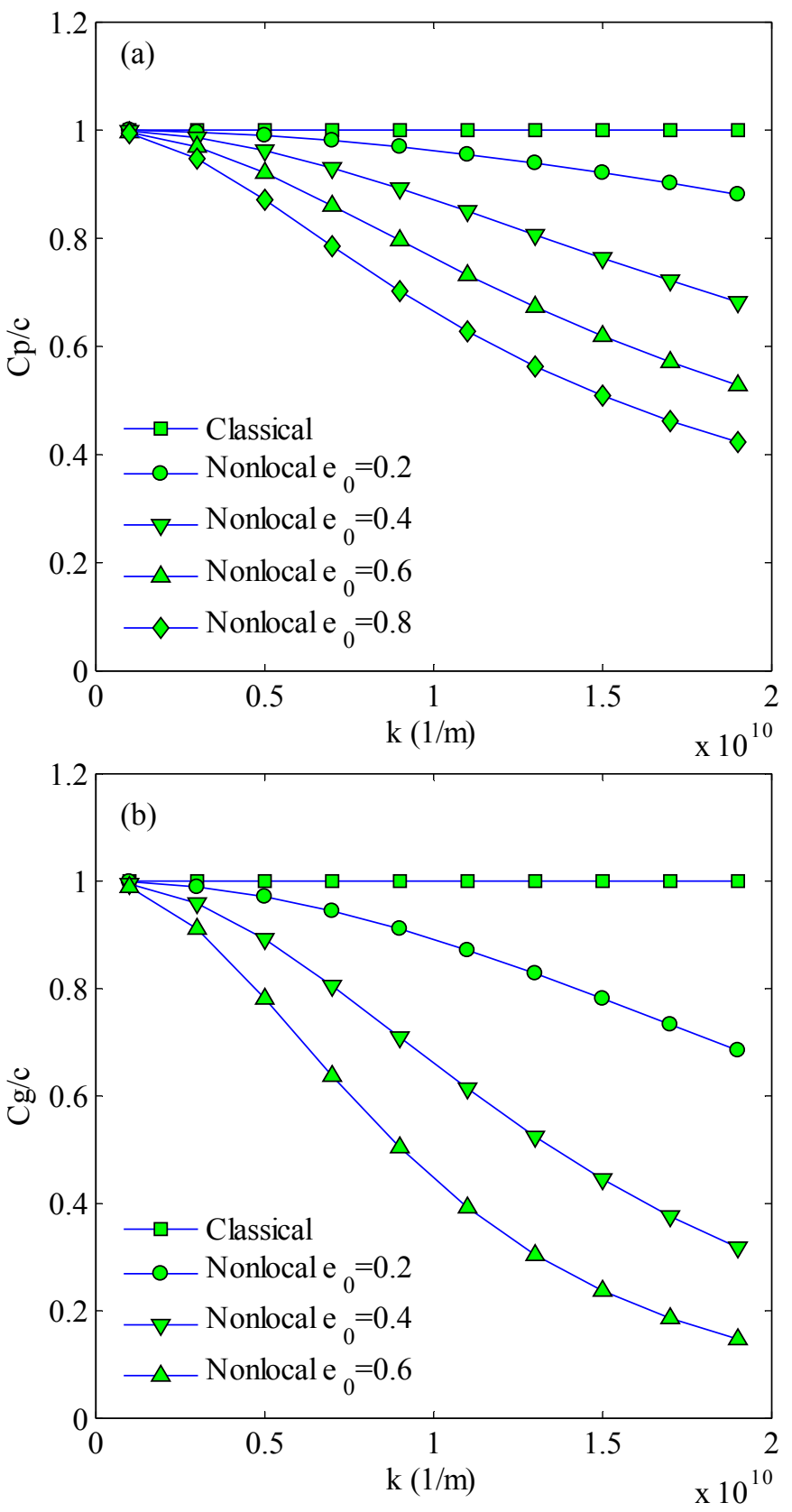

Fig. 2. Comparison of classical and nonlocal models at different wave-numbers and for different values of the nonlocal constant $e_{0}$ : (a) phase velocities (b) group velocities. The dispersive behavior is clearly seen in the nonlocal model results, whereas the classical model predicts a non-dispersive behavior.

The above comparisons can be better understood using a graphical demonstration. Fig. 2 (a) compares classical and nonlocal models in terms of the ratio of phase velocity to the shear speed of sound for various values of the wave-number and different values of the nonlocal constant, $e_{0}$. Fig. 2 (b) shows the same comparison for group velocities. The dispersive behavior of torsional waves in the nonlocal model and their non-dispersive behavior in the classical model are clearly observed in Fig. 2.

\section{MD SimULATIONS}

The MD simulation technique using NanoHive-1 software [18] is used to simulate free torsional vibrations on $(6,6)$ and $(10,10)$ armchair nanotubes. The simulations are based on the Adaptive Intermolecular Reactive Empirical Bond Order (AIREBO) potential [19] which is widely used for simulating CNTs. Different torsional natural mode-shapes of each nanotube are simulated and the corresponding natural frequencies are extracted using the simulation outputs.

\section{A. Simulation Methodology and Setup}

To find the natural frequency corresponding to each mode-shape the nanotube is pre-deformed to that mode-shape by mathematically changing the coordinates (Fig. 3) and rotating each row of atoms around the CNT axis according to the following relation:

$\phi(x, 0)=A \sin \frac{k^{\prime} \pi x}{l}$,

where $k^{\prime}$ is the mode-number (or half wave-length) corresponding to a certain mode-shape and $l$ is the length of the CNT. Then the atoms at both ends are fixed to simulate pined-pined boundary conditions (Fig. 3) and the CNT is allowed to vibrate freely for 100,000 time steps of $0.5 \mathrm{fs}$.

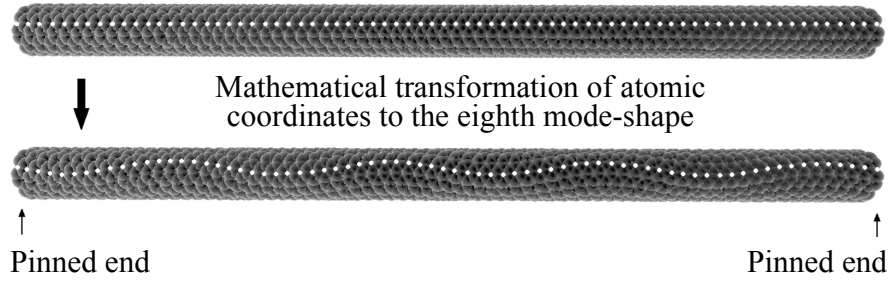

Fig. 3. Free torsional vibrations of a $(6,6)$ armchair CNT at the eighth natural mode-shape corresponding to $k^{\prime}=8$, where the mode-shape is induced by mathematically changing the atomic coordinates initially.

The rotation of the cross-sections of the CNT is extracted as an output of the simulation and plotted against time using MATLAB software. The natural vibration period is extracted by visualizing the free vibrations in the NanoHive-1 post processor. The time period is then used to find the natural frequency of vibration corresponding to that mode-shape. As an example, Fig. 4 shows the free torsional vibrations of a $(6,6)$ armchair CNT in the eighth natural modes-shape corresponding to $k^{\prime}=8$. At $t=0$ the simulation starts and the nanotube is allowed to undergo free torsional vibrations. The rotation of the cross-sections of the CNT at different instances is shown. It is evident that the 8 th mode-shape has a vibration period of $\tau=320 \mathrm{fs}$.

An important consideration when performing the above molecular dynamics simulations is the role of the thermostat. By scaling the velocities to keep the temperature constant, the thermostat is randomly introducing or taking away kinetic energy to or from the system. Furthermore, the thermostatic kinetic energy is divided between atoms randomly and might 
excite mode-shapes other than the original mode-shape of the simulation. The purpose of the above simulations is to find the natural frequencies of pure mode-shapes in free torsional vibrations, and thus the thermostat which acts as an external stimulus is not applied to the system. Omitting thermostatic effects, the only change in kinetic energy is due to the conversion of potential energy of vibration and vice versa, and the total energy of the system remains constant. Thus the present simulations pertain to free, undamped torsional vibrations.

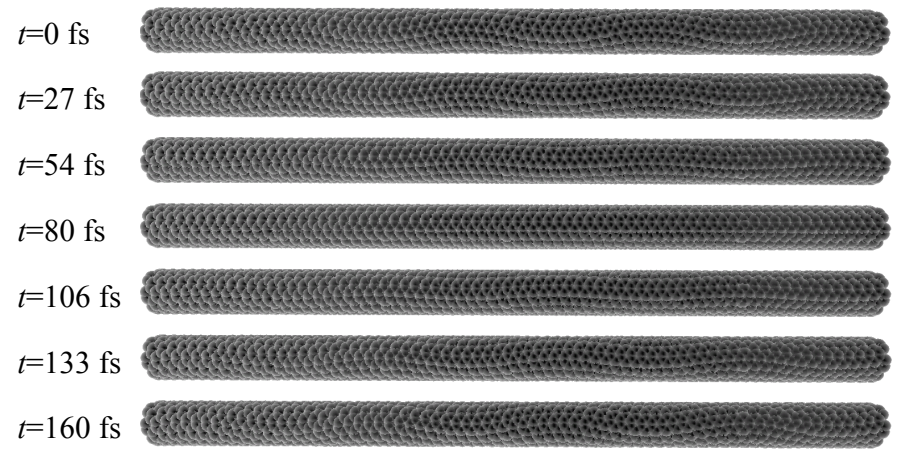

Fig. 4- Free torsional vibrations of a $(6,6)$ armchair $\mathrm{CNT}$ at the $8^{\text {th }}$ natural mode-shape corresponding to $k^{\prime}=8$. Different instances of half a vibration period $(\tau / 2=160 \mathrm{fs})$ are shown.

The lengths of the CNTs were chosen such that each CNT has 145 rows of carbon atoms in the axial direction. The number of rows chosen above has the benefit that most of the simulated mode-shapes have nodes that coincide with the spatial position (longitudinal coordinate) of a certain row of atoms. Since the axial distance between two consecutive rows in an armchair nanotube is 1.21 angstroms, the total length of each simulated nanotube is 174.24 angstroms.

\section{B. Simulation Results}

Results from MD simulation for the vibration periods and natural frequencies of the different mode-shapes of $(6,6)$ and $(10,10)$ armchair nanotubes are shown in Tables I and II, respectively.

From the data in Tables I and II, it is seen that the vibration frequencies of the simulated pinned-pinned nanotubes are generally in the terahertz range. For the same mode-shape or wave-length the $(6,6)$ armchair CNT has slightly higher vibration frequencies compared to the $(10,10)$ armchair, which is due to the slightly higher surface shear modulus of the $(6,6)$ CNT. A comparison of the data obtained for the first and last mode-shapes of each of the simulated nanotubes shows that the torsional vibrations of the CNTs do not follow the classical dispersion relations of equation (6). This observation is further investigated in section $C$.
TABLE I

Vibration Periods and Natural FREQUencies of SEVERAL DifFERENT MODE-SHAPES OF A $(6,6)$ ARMCHAIR CNT

\begin{tabular}{ccccc}
\hline \hline $\begin{array}{c}\text { Mode-shape } \\
k^{\prime}\end{array}$ & $\begin{array}{c}\text { Wavelength } \\
\lambda(\mathrm{m})\end{array}$ & $\begin{array}{c}\text { Period } \\
\tau(\mathrm{s})\end{array}$ & $\begin{array}{c}\text { Frequency } \\
f(\mathrm{~Hz})\end{array}$ & $\begin{array}{c}\text { Angular Freq. } \\
\omega(\mathrm{rad} / \mathrm{s})\end{array}$ \\
\hline 1 & $3.50 \mathrm{E}-08$ & $2.52 \mathrm{E}-12$ & $3.97 \mathrm{E}+11$ & $2.50 \mathrm{E}+12$ \\
2 & $1.75 \mathrm{E}-08$ & $1.26 \mathrm{E}-12$ & $7.92 \mathrm{E}+11$ & $4.97 \mathrm{E}+12$ \\
4 & $8.75 \mathrm{E}-09$ & $6.36 \mathrm{E}-13$ & $1.57 \mathrm{E}+12$ & $9.88 \mathrm{E}+12$ \\
8 & $4.38 \mathrm{E}-09$ & $3.20 \mathrm{E}-13$ & $3.13 \mathrm{E}+12$ & $1.96 \mathrm{E}+13$ \\
9 & $3.89 \mathrm{E}-09$ & $2.86 \mathrm{E}-13$ & $3.50 \mathrm{E}+12$ & $2.20 \mathrm{E}+13$ \\
12 & $2.92 \mathrm{E}-09$ & $2.10 \mathrm{E}-13$ & $4.76 \mathrm{E}+12$ & $2.99 \mathrm{E}+13$ \\
16 & $2.19 \mathrm{E}-09$ & $1.60 \mathrm{E}-13$ & $6.27 \mathrm{E}+12$ & $3.94 \mathrm{E}+13$ \\
18 & $1.94 \mathrm{E}-09$ & $1.41 \mathrm{E}-13$ & $7.09 \mathrm{E}+12$ & $4.46 \mathrm{E}+13$ \\
24 & $1.46 \mathrm{E}-09$ & $1.07 \mathrm{E}-13$ & $9.39 \mathrm{E}+12$ & $5.90 \mathrm{E}+13$ \\
36 & $9.72 \mathrm{E}-10$ & $7.10 \mathrm{E}-14$ & $1.41 \mathrm{E}+13$ & $8.85 \mathrm{E}+13$ \\
48 & $7.29 \mathrm{E}-10$ & $5.40 \mathrm{E}-14$ & $1.85 \mathrm{E}+13$ & $1.16 \mathrm{E}+14$ \\
54 & $6.48 \mathrm{E}-10$ & $4.80 \mathrm{E}-14$ & $2.08 \mathrm{E}+13$ & $1.31 \mathrm{E}+14$ \\
66 & $5.30 \mathrm{E}-10$ & $4.00 \mathrm{E}-14$ & $2.50 \mathrm{E}+13$ & $1.57 \mathrm{E}+14$ \\
72 & $4.86 \mathrm{E}-10$ & $3.68 \mathrm{E}-14$ & $2.72 \mathrm{E}+13$ & $1.71 \mathrm{E}+14$ \\
\hline \hline
\end{tabular}

TABLE II

VIBRATION PERIODS AND NATURAL FREQUENCIES OF SEVERAL DifFERENT MODE-SHAPES OF A $(10,10)$ ARMCHAIR CNT

\begin{tabular}{ccccc}
\hline $\begin{array}{c}\text { Mode-shape } \\
k^{\prime}\end{array}$ & $\begin{array}{c}\text { Wavelength } \\
\lambda(\mathrm{m})\end{array}$ & $\begin{array}{c}\text { Period } \\
\tau(\mathrm{s})\end{array}$ & $\begin{array}{c}\text { Frequency } \\
f(\mathrm{~Hz})\end{array}$ & $\begin{array}{c}\text { Angular Freq. } \\
\omega(\mathrm{rad} / \mathrm{s})\end{array}$ \\
\hline 1 & $3.50 \mathrm{E}-08$ & $2.61 \mathrm{E}-12$ & $3.83 \mathrm{E}+11$ & $2.41 \mathrm{E}+12$ \\
4 & $8.75 \mathrm{E}-09$ & $6.53 \mathrm{E}-13$ & $1.53 \mathrm{E}+12$ & $9.62 \mathrm{E}+12$ \\
16 & $2.19 \mathrm{E}-09$ & $1.64 \mathrm{E}-13$ & $6.11 \mathrm{E}+12$ & $3.84 \mathrm{E}+13$ \\
24 & $1.46 \mathrm{E}-09$ & $1.09 \mathrm{E}-13$ & $9.17 \mathrm{E}+12$ & $5.76 \mathrm{E}+13$ \\
36 & $9.72 \mathrm{E}-10$ & $7.37 \mathrm{E}-14$ & $1.36 \mathrm{E}+13$ & $8.53 \mathrm{E}+13$ \\
54 & $6.48 \mathrm{E}-10$ & $5.00 \mathrm{E}-14$ & $2.00 \mathrm{E}+13$ & $1.26 \mathrm{E}+14$ \\
66 & $5.30 \mathrm{E}-10$ & $4.15 \mathrm{E}-14$ & $2.41 \mathrm{E}+13$ & $1.51 \mathrm{E}+14$ \\
72 & $4.86 \mathrm{E}-10$ & $3.80 \mathrm{E}-14$ & $2.63 \mathrm{E}+13$ & $1.65 \mathrm{E}+14$ \\
\hline \hline
\end{tabular}

\section{Comparison of MD Simulation Results with Classical and Nonlocal Models}

Both the classical and nonlocal models for the angular frequency of torsional vibrations, seen in equations (14) and (13), respectively, are dependent on the value of the shear speed of sound $(c)$ in CNTs, which is equal to:

$c=\sqrt{\frac{G}{\rho}}=\sqrt{\frac{G \cdot h}{\rho . h}}$,

where $G . h$ is the surface shear modulus previously obtained by the authors [19], $\rho$ is the mass density of the CNT and $\rho . h$ is the surface mass density found using the following relation:

$\rho=\frac{m}{V}=\frac{m}{2 \pi a h l} \Rightarrow \rho \cdot h=\frac{m}{2 \pi a l}$.

Thus, knowing the number of carbon atoms in the CNT, the mass of each carbon atom and the radius, $a$, and length, $l$, of the CNT, the surface mass density, $\rho . h$, is easily calculated and used in equation (19) along with the value of the corresponding surface shear modulus, G.h, to find the shear speed of sound, $c$. 
The values of the shear speed of sound calculated for $(6,6)$ and $(10,10)$ armchair nanotubes using the above approach are shown in Table III. Due to a slightly higher stiffness, the $(6,6)$ nanotube has a slightly higher shear speed of sound compared to the $(10,10)$ nanotube.

TABLE III

Surface Shear Modulus, Surface Density and Shear Speed of Sound FOR $(6,6)$ AND $(10,10)$ ARMCHAIR CNTS

\begin{tabular}{cccc}
\hline \hline $\mathrm{CNT}$ & $G . h(\mathrm{~N} / \mathrm{m})$ & $\rho . h\left(\mathrm{~kg} / \mathrm{m}^{2}\right)$ & $c(\mathrm{~m} / \mathrm{s})$ \\
\hline$(6,6)$ & 153.7 & $7.88 \mathrm{E}-7$ & $1.39 \mathrm{E} 4$ \\
$(10,10)$ & 145.3 & $7.88 \mathrm{E}-7$ & $1.35 \mathrm{E} 4$ \\
\hline \hline
\end{tabular}

\section{Comparison of angular frequencies}

Based on the classical model of equation (14) and the values of sound speed given in Table III, the angular frequencies of the first mode-shape $\left(k^{\prime}=1\right)$ of torsional vibrations for $(6,6)$ and $(10,10)$ armchair CNTs are:

$$
\begin{aligned}
& (6,6) \rightarrow \omega_{k^{\prime}=1}=2.51 \times 10^{12} \quad(\mathrm{rad} / \mathrm{s}) \\
& (10,10) \rightarrow \omega_{k^{\prime}=1}=2.42 \times 10^{12} \quad(\mathrm{rad} / \mathrm{s})
\end{aligned}
$$

These values are almost identical to the angular frequencies obtained from molecular dynamics shown in Tables I and II (the error is less than $0.5 \%$ ). If the same classical model is used to find the angular frequencies of the $72^{\text {nd }}$ mode-shape $\left(k^{\prime}=72\right)$ of torsional vibrations for $(6,6)$ and $(10,10)$ armchair CNTs the following values are obtained.

$$
\begin{aligned}
& (6,6) \rightarrow \omega_{k^{\prime}=72}=1.80 \times 10^{14} \quad(\mathrm{rad} / \mathrm{s}) \\
& (10,10) \rightarrow \omega_{k^{\prime}=72}=1.74 \times 10^{14}(\mathrm{rad} / \mathrm{s})
\end{aligned}
$$

The angular frequency values obtained from the classical model are higher than those of MD simulation results (shown in Tables I and II) and have errors of more than 5\%. It is evident that at these higher mode-shapes with smaller wavelengths, the classical model loses its accuracy (Fig. 5), as discussed in section 2.3.

In comparison, in the nonlocal model of equation (13) the term in the denominator grows with increasing values of wave-number and could compensate for the size effects that become significant at smaller wavelengths. A least square optimization algorithm is used to find the correct value of the nonlocal parameter $\xi$ (or nonlocal constant $e_{0}$ ) to be used in the nonlocal model. The optimized values for the nonlocal constant are found as 0.185 and 0.18 for the $(6,6$,$) and (10,10)$ nanotubes, respectively. It is seen that using these nonlocal constant values the nonlocal model can predict the MD simulation results with sufficient accuracy, both at small and large wavelengths (Fig. 5). The above results obtained for the nonlocal constant values are comparable to those previously reported. Hu et al. [14] compared nonlocal shell models for dispersion of torsional waves in CNTs with results from molecular dynamics simulations and reported an $e_{0}$ value of 0.2 for $(10,10)$ armchair CNTs.

A question of practical interest is whether the nonlocal parameter, $\xi=\left(e_{0} d\right)^{2}$, is unique? Currently available results from the literature suggest otherwise [13, 15, 16, 20]. A primary reason for this is the chirality of a nanotube which introduces an effect similar to anisotropy in an equivalent continuum structure. It is easy to visualize this behavior if one treats a nanotube as a cellular structure and experimentally observes its response for different chiralities. Another consequence of chirality is that equivalent continuum elastic properties would also depend on the mode of loading. It is therefore valuable to develop a relationship between the chirality and nonlocal parameter for different modes of loading through either theoretical or numerical simulation studies.
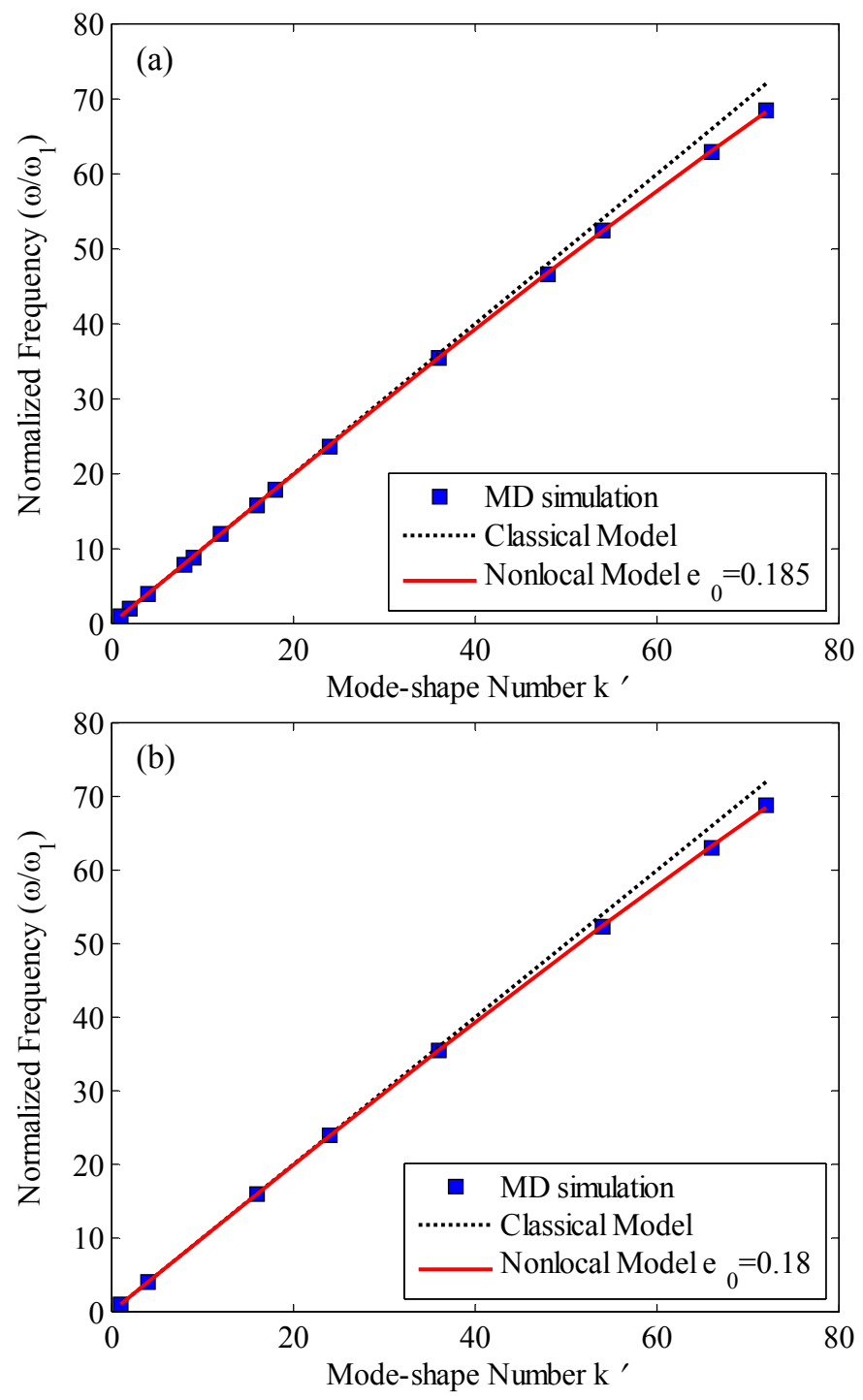

Fig. 5. Comparison of classical and nonlocal models for the normalized angular frequency versus mode-shape number with MD simulation results (a) $(6,6)$ CNT (b) $(10,10)$ CNT. For each nanotube the corresponding nonlocal constant to obtain the best fit is shown.

\section{Comparison of group velocities}

The group velocity is the slope of the natural frequency versus wave-number $(\omega-k)$ curve. A numerical differentiation technique can be used to find the slope, such as a two-point 
numerical differentiation of the form:

slope at wavenumber $k=\frac{\omega_{k}-\omega_{k-1}}{k-(k-1)}$.

When using the above numerical differentiation technique, small errors in reading the MD simulation output can lead to large errors in the derivative. A better way of finding the slope would be to use curve-fitting to find a polynomial that portrays the general behavior of the $\omega-k$ curve and then use analytical differentiation to find the derivative of the polynomial and calculate its value at different wave-numbers. As the nonlocal model gives a precise fit to MD simulations, a polynomial based on the nonlocal model could accurately model the MD $\omega-k$ behavior.

Based on equation (15) and the discussion presented in section 2.3, different polynomials are fit to the $\omega-k$ curve and it is seen that a third-order polynomial $\left(\omega_{(k)}=a k+b k^{3}\right)$ can model the $\omega-k$ behavior with high accuracy. This third-order polynomial is then differentiated to find the group velocities at different wave-numbers. The MD group velocities obtained for $(6,6)$ and $(10,10)$ armchair nanotubes are presented in Fig. 6 and compared with the group velocities obtained from the classical and nonlocal models of equations (14) and (13), respectively.

The classical model predicts a constant group velocity equal to the shear speed of sound, $c$. The nonlocal model predicts group velocities that decrease in a nonlinear manner with the increase of the corresponding wave-numbers. Once again, it is seen that the classical model is accurate for smaller wave-numbers (larger wavelengths) but loses its accuracy at higher wave-numbers. If the classical model is used for higher wave-numbers it can result in errors of more than $15 \%$ when compared to MD simulations (Fig. 6). In contrast to the classical model, the nonlocal model is accurate at all wave-numbers (wavelengths) and, when used along with the nonlocal constants derived in section 3.3, it reproduces the dispersion behavior seen in MD simulations. Fig. 6 compares the classical and nonlocal models for group velocity with the values obtained from MD simulations.

It is interesting to see how well the nonlocal model can predict the group velocities obtained from MD simulations. This is done by studying the "goodness of fit" in Fig. 6 through the chi-square $\left(\chi^{2}\right)$ test. The chi-square parameter is the sum of the differences between observed and expected outcomes, each squared and divided by the expectation:

$\chi^{2}=\sum \frac{(O-E)^{2}}{E}$

In the current example, the observed quantities are the MD simulation results and the expected outcomes are the results from the nonlocal model. Fig. 6 (a) and Fig. 6 (b) have $\chi^{2}$ values of $1.49 \mathrm{e}-4$ and $2.26 \mathrm{e}-4$, respectively. The $\chi^{2}$ values are very small compared to the average value of the observed or expected outcome (which is between 0.84 and 1), and this indicates a very high goodness of fit.
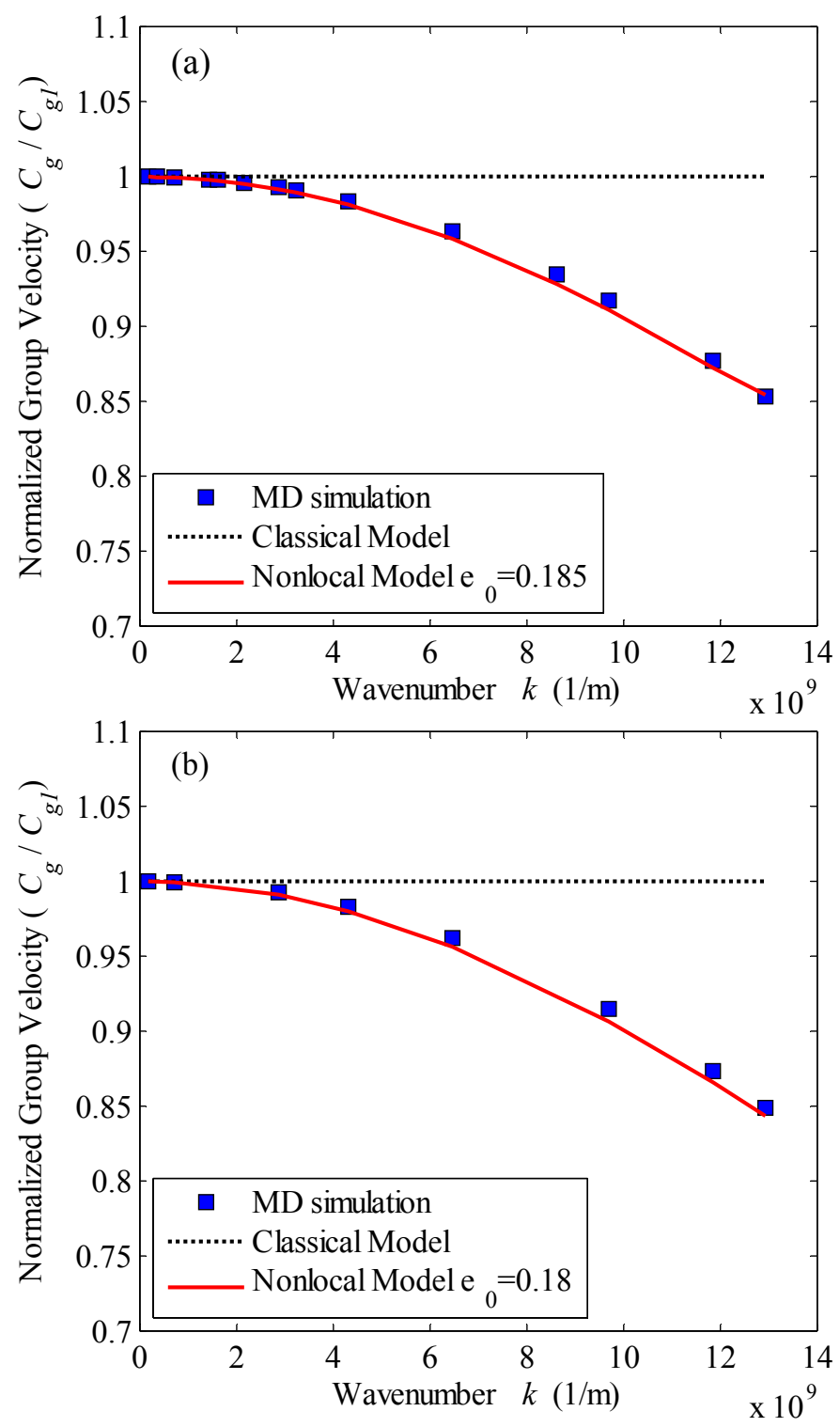

Fig. 6. Comparison of classical and nonlocal models for the normalized group velocity versus wave-number with MD simulation results for (a) $(6,6)$ CNT (b) $(10,10) \mathrm{CNT}$.

\section{Comparison of phase velocities}

The phase velocities corresponding to MD simulations can easily be found by finding the ratios of the natural frequencies $\omega$ to their corresponding wave-numbers $k$. The polynomial expression found in the previous section describing the $\omega-k$ behavior of MD simulations is used to find the $\omega / k$ ratio and calculate the phase velocity corresponding to each wave-number $k$. The phase velocity values of the different mode shapes of $(6,6)$ and $(10,10)$ armchair CNTs obtained from molecular dynamics are shown in Fig. 7 along with the phase velocities calculated using the classical and nonlocal models (equations (6) and (7)).

The classical model predicts a constant phase velocity equal 
to the shear speed of sound $c$. The nonlocal model predicts phase velocities that decrease in a nonlinear manner with the increase of the corresponding wave-numbers. Once again it is seen that the classical model is accurate for smaller wave-numbers (larger wavelengths) but loses its accuracy at higher wave-numbers. In contrast, the nonlocal model is accurate at all wave-numbers (wavelengths) and, when used along with the nonlocal constants derived in section 3.3, it can precisely convey the dispersion behavior obtained from MD simulations.
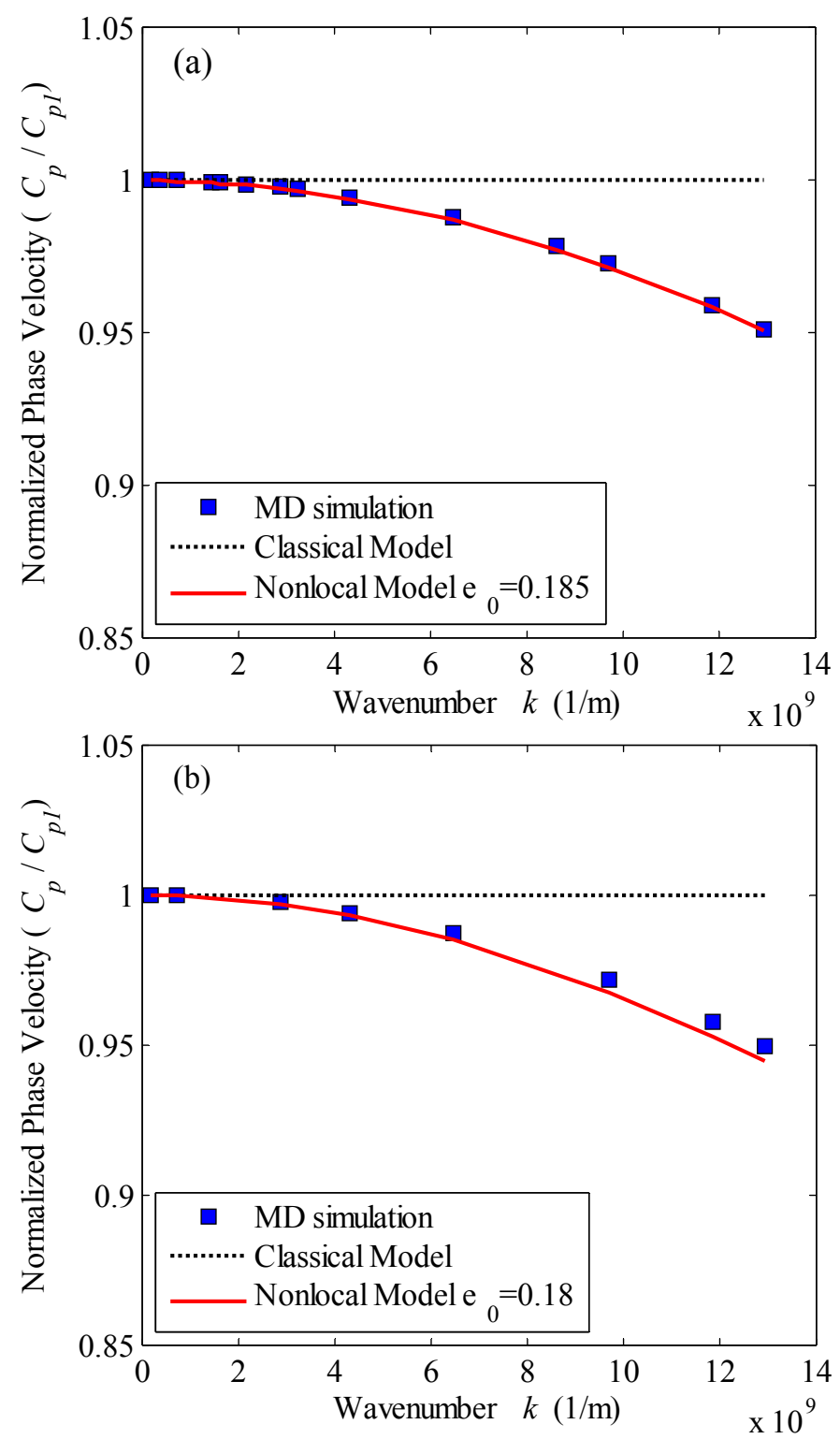

Fig. 7. Comparison of local and nonlocal models for the normalized phase velocity versus wave-number with MD simulation results. (a) $(6,6) \mathrm{CNT}$ (b) $(10,10)$ CNT

It is important to note that the dispersion behavior of phase velocities seen in the nonlocal model as well as molecular dynamics simulations (Fig. 7) is similar to the behavior previously reported in [16]. However, the phase velocity derived for the first mode-shape in the present work (which is equal to the shear speed of sound) is larger than the value previously reported [16]. This is probably due to the higher torsional stiffness or surface shear moduli that has been derived for the armchair nanotubes in the present work, which in turn could be caused by the use of different potential functions in the MD simulations and the simulation temperatures. Once again, we emphasize that the approach used for finding the value of the nonlocal constant in the present work is more accurate compared to that of [16] due to the large number of data points simulated in the high frequency region of the dispersion curves.

Regarding the accuracy of the nonlocal model in predicting the MD simulation results for phase velocities, Fig. 7 (a) and Fig. 7 (b) have chi-square parameter $\left(\chi^{2}\right)$ values of $6.94 \mathrm{e}-6$ and $7.457 \mathrm{e}-5$, respectively, which once again indicate a very high degree of fit of the nonlocal model to the MD simulation results.

A lumped parameter model can be postulated for the paddle oscillator in Fig. 1. The CNTs on either side of the block can be modeled as two torsional springs in parallel, each of stiffness $G_{\text {eff. }} I_{p} / L$, where $G_{\text {eff }}$ is the effective nonlocal shear modulus, $I_{p}$ is the polar area moment of inertia of cross section, and $L$ is the length of the CNT element. The effective shear modulus $G_{\text {eff }}$ can be obtained from a comparison of the classical dispersion relation for shear waves $\left(\omega^{2}=c^{2} k^{2}\right)$ with the corresponding dispersion relation of the nonlocal continuum model given in equation (5): $G_{\text {eff }}=G /\left(1+\xi k^{2}\right)$. The equivalent mass moment of inertia from the central block and the CNT springs, $J_{\text {total }}$ can be found for each mode. It may be observed that the effective torsional stiffness of oscillator depends on the nonlocal parameter $\xi$ and the wave-number, $k$, via the effective shear modulus: the effective shear modulus of the nanotube, $G_{\text {eff }}$, decreases with increasing wave-number, $k$, indicating that nonlocal effects are significant for short wavelengths deformation modes, or, high frequency modes of operation of the resonator. One should also note that increasing the central mass increases $J_{\text {total }}$, and hence decreases the natural frequencies.

\section{CONCLUSIONS}

MD simulation of torsional vibrations of a pinned-pinned CNT provides a simple, effective and efficient method for finding the natural frequency of vibrations. The natural frequency can subsequently be used to find the group and phase velocities of torsional waves propagating in a CNT. Through comparison of the MD simulation results with classical and nonlocal dispersion relations, it is found that the nonlocal constant is about 0.18 for torsional waves propagating in $(6,6)$ and $(10,10)$ armchair nanotubes. Using this value for the nonlocal constant, the nonlocal dispersion relation can predict the MD natural frequencies, group and phase velocities with high accuracy (maximum error of $0.5 \%$ ). Use of the classical dispersion relations could lead to errors as high as $15 \%$ in predicting the group velocity of torsional waves in the high frequency region. Taking into account that the group velocity governs the flow of energy in a system, this error could be a 
significant factor in the design of devices such as torsional oscillators or electro-mechanical delay elements based on CNT torsion, and has to be accounted for. The superiority of the nonlocal model over the classical model in predicting the real dispersion behavior of torsional waves in CNTs is established.

\section{REFERENCES}

[1] S. J. Papadakis, A. R. Hall, P. A. Williams, L. Vicci, M. R. Falvo, R. Superfine and S. Washburn, "Resonant oscillators with carbon-nanotube torsion springs," Phys. Rev. Lett., vol. 93, pp. 146101-146101, 2004.

[2] A. R. Hall, L. An, J. Liu, L. Vicci, M. R. Falvo, R. Superfine and S. Washburn, "Experimental Measurement of Single-Wall Carbon Nanotube Torsional Properties," Phys. Rev. Lett., vol. 96, pp. 256102, 2006.

[3] A. R. Hall, M. R. Falvo, R. Superfine and S. Washburn, "A Self-Sensing Nanomechanical Resonator Built on a Single-Walled Carbon Nanotube," Nano Letters, vol. 8, pp. 3746-3749, 2008.

[4] S. Xiao and W. Hou, "Studies of nanotube-based resonant oscillators through multiscale modeling and simulation," Physical Review B, vol. 75, pp. $125414,2007$.

[5] S. Xiao and W. Hou, "Multiscale modeling and simulation of nanotube-based torsional oscillators," Nanoscale Research Letters, vol. 2, pp. 54-59, 2007.

[6] B. Yakobson, C. Brabec and J. Bernholc, "Nanomechanics of Carbon Tubes: Instabilities beyond Linear Response," Phys. Rev. Lett., vol. 76, pp. 2511-2514, 1996

[7] G. Zhou, W. Duan and B. Gu, "First-principles study on morphology and mechanical properties of single-walled carbon nanotube," Chem. Phys. Lett., vol. 333, pp. 344-349, 2001.

[8] M. A. L. Marques, H. E. Troiani, M. Miki-Yoshida, M. Jose-Yacaman and A. Rubio, "On the Breaking of Carbon Nanotubes under Tension," Nano Letters, vol. 4, pp. 811-816, 2004.

[9] A. C. Eringen, "Linear theory of nonlocal elasticity and dispersion of plane waves," Int. J. Eng. Sci., vol. 10, pp. 425-435, 1972.

[10] J. Peddieson, G. R. Buchanan and R. P. McNitt, "Application of nonlocal continuum models to nanotechnology," Int. J. Eng. Sci., vol. 41, pp. 305-312, 2003.

[11] C. M. Wang, Y. Y. Zhang and X. Q. He, "Vibration of nonlocal Timoshenko beams," Nanotechnology, vol. 18, pp. 105401, 2007.

[12] Q. Wang and V. K. Varadan, "Application of nonlocal elastic shell theory in wave propagation analysis of carbon nanotubes," Smart Mater. Struct., vol. 16, pp. 178-190, 2007.

[13] Y. Q. Zhang, G. R. Liu and X. Y. Xie, "Free transverse vibrations of double-walled carbon nanotubes using a theory of nonlocal elasticity," Phys. Rev. B, vol. 71, pp. 195404, 2005.

[14] M. Xu, "Free transverse vibrations of nano-to-micron scale beams," Proceedings of the Royal Society A: Mathematical, Physical and Engineering Sciences, vol. 462, pp. 2977-2995, 2006.

[15] Q. Wang, "Wave propagation in carbon nanotubes via nonlocal continuum mechanics," J. Appl. Phys., vol. 98, pp. 124301, 2005.

[16] Y. G. Hu, K. Liew, Q. Wang, X. He and B. Yakobson, "Nonlocal shell model for elastic wave propagation in single-and double-walled carbon nanotubes," J. Mech. Phys. Solids, vol. 56, pp. 3475-3485, 2008.

[17] S. Timoshenko and J. M. Gere, Theory of Elastic Stability. New York, USA: McGraw-Hill, 1961, pp. 452-509.

[18] NanoHive-1, v.1.2.0-b1., Nanorex Inc., www.nanorex.com, 2005.

[19] S. J. Stuart, A. B. Tutein and J. A. Harrison, "A reactive potential for hydrocarbons with intermolecular interactions," J. Chem. Phys., vol. 112, pp. 6472-6486, 2000.

[20] F. Khademolhosseini, "Nonlocal continuum shell models for torsion of single-walled carbon nanotubes" M.Sc. thesis, Dept. Mech. Eng., Univ. of British Columbia, Vancouver, Canada, 2009.

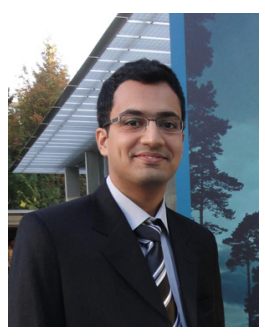

Farzad Khademolhosseini received his Bachelor's degree in Mechanical Engineering from Sharif University of Technology, Tehran, Iran, in 2007. He then joined the department of Mechanical Engineering at the University of British Columbia (UBC) and the UBC Microsystems and Nanotechnology group (MiNa) were he conducted research in the field of nanomechanics, molecular and atomistic modeling and obtained a Master's degree in Mechanical Engineering in 2009.

Since 2009 he has been working towards a Ph.D. degree in the department of Mechanical Engineering at UBC. His current research interests include biomedical microdevices and modeling of cell-mechanics.

$\mathrm{He}$ is a Fellow of the Killam Foundation and the recipient of multiple research awards including the Killam Doctoral Fellowship and the UBC Four Year Doctoral Fellowship.

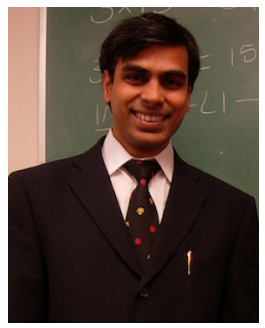

A. Srikantha Phani received the B.Tech. (First Class Honors with Distinction) in civil engineering from Nagarjuna University, Guntur, India, in 1998, the M.Sc. degree in aerospace engineering from Indian Institute of Science, Bangalore, India, in 2000, and the Ph.D. degree from University of Cambridge, Cambridge, U.K., in 2004.

$\mathrm{He}$ is currently an Assistant Professor in the Department of Mechanical Engineering, The University of British Columbia, Vancouver, B.C., Canada. His research interests are in applied mechanic; dynamics of lattice materials and devices.

Dr. Phani is a Tier 2 Canada Research Chair in Dynamics of Lattice Materials and Devices. He is a Fellow of Cambridge Commonwealth Society.

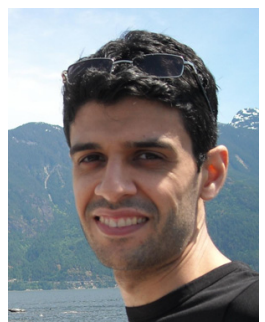

Alireza Nojeh is a member of the IEEE. He received his B.S. and M. S. degrees in electrical engineering from Sharif University of Technology, Tehran, Iran in 1997 and 1999, respectively. He went on to receive a D.E.A. degree in electronics/optoelectronics from the University of Paris XI, Orsay, France in 2000 and a $\mathrm{Ph} . \mathrm{D}$. degree in electrical engineering from Stanford University, Stanford, California, USA in 2006. He has been an assistant professor of electrical and computer engineering at the University of British

Columbia since 2006

His research interests are in nanotechnology, in particular on carbon nanotube devices; electron sources, vacuum electronics and electron microscopy; micro/nanofabrication; modeling and simulation of nanoscale structures; solid-state electronics and optoelectronics.

Dr. Nojeh is also a member of the American Vacuum Society and the Materials Research Society.

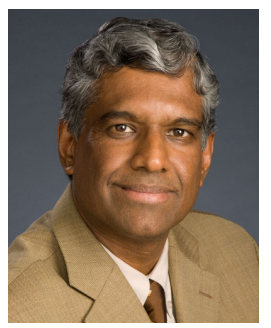

Nimal Rajapakse received his B.Sc. (Honours) in Civil Engineering from the University of Sri Lanka in 1977 and M.Eng. and D.Eng. degrees from the Asian Institute of Technology in 1981 and 1983 respectively.

He is currently the Dean of the Faculty of Applied Sciences at Simon Fraser University and a Professor in the School of Engineering Science. His research interests cover nanomechanics, computational mechanics and solid mechanics.

He is a Fellow of the Canadian Academy of Engineering and the Engineering Institute of Canada 